ARTICLE

\title{
Large-area synthesis of nanoscopic catalyst-decorated conductive MOF film using microfluidic-based solution shearing
}

\author{
Jin-Oh Kim (1) 1,5, Won-Tae Koo ${ }^{1,2,5}$, Hanul Kim (10 3 ${ }^{3}$, Chungseong Park'1,2, Taehoon Lee (1) 1,

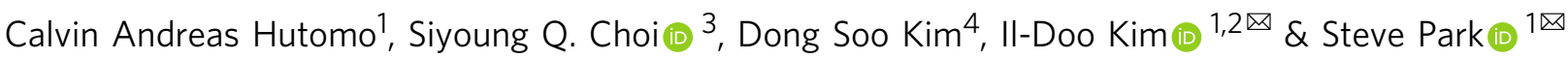

Conductive metal-organic framework (C-MOF) thin-films have a wide variety of potential applications in the field of electronics, sensors, and energy devices. The immobilization of various functional species within the pores of $\mathrm{C}$-MOFs can further improve the performance and extend the potential applications of C-MOFs thin films. However, developing facile and scalable synthesis of high quality ultra-thin C-MOFs while simultaneously immobilizing functional species within the MOF pores remains challenging. Here, we develop microfluidic channel-embedded solution-shearing (MiCS) for ultra-fast $(\leq 5 \mathrm{~mm} / \mathrm{s})$ and large-area synthesis of high quality nanocatalyst-embedded C-MOF thin films with thickness controllability down to tens of nanometers. The MiCS method synthesizes nanoscopic catalystembedded C-MOF particles within the microfluidic channels, and simultaneously grows catalyst-embedded C-MOF thin-film uniformly over a large area using solution shearing. The thin film displays high nitrogen dioxide $\left(\mathrm{NO}_{2}\right)$ sensing properties at room temperature in air amongst two-dimensional materials, owing to the high surface area and porosity of the ultrathin C-MOFs, and the catalytic activity of the nanoscopic catalysts embedded in the C-MOFs. Therefore, our method, i.e. MiCS, can provide an efficient way to fabricate highly active and conductive porous materials for various applications.

\footnotetext{
${ }^{1}$ Department of Materials Science and Engineering, Korea Advanced Institute of Science and Technology (KAIST), Daejeon, Republic of Korea. ${ }^{2}$ Membrane Innovation Center for Anti-virus \& Air-quality Control, KAIST Institute for Nanocentury, Daejeon, Republic of Korea. ${ }^{3}$ Department of Chemical and Biomolecular Engineering, Korea Advanced Institute of Science and Technology (KAIST), Daejeon, Korea. ${ }^{4}$ Department of Creative Convergence Engineering, Hanbat National University, Daejeon, Korea. ${ }^{5}$ These authors contributed equally: Jin-Oh Kim, Won-Tae Koo. ${ }^{凶}$ email: idkim@kaist.ac.kr; stevepark@kaist.ac.kr
} 
C onductive metal-organic frameworks (C-MOFs) are emerging materials receiving a great deal of interest in recent years due to their many attractive properties, such as high porosity, narrow pore-size distribution and periodically organized pores, adjustable bandgap, and designable electrical charge transport properties ${ }^{1-5}$. These properties broaden the applicability of metal-organic frameworks (MOFs) (which have traditionally been electrically insulating) to various applications such as transistors ${ }^{6}$, electrodes ${ }^{7-10}$, and resistive chemical sensors $^{11-14}$.

The key features of MOFs are their high porosity and regularly arranged pores. These pores can be utilized to immobilize nanoscale catalysts such as $\mathrm{Au}, \mathrm{Pd}$, and $\mathrm{Pt}$, and since the reactivity of the catalysts improves with increasing surface area, welldispersed nanoscale catalysts can drastically enhance the overall catalytic performance of MOFs ${ }^{15-19}$. Recently, the immobilization of nanocatalyst has been applied to C-MOFs, and was demonstrated of enhancing the performance of $\mathrm{Li}-\mathrm{S}$ batteries ${ }^{20}$ and gas sensors ${ }^{15}$.

The C-MOF applications mentioned above require the formation of high-quality thin films with controlled film thickness down to nanometer scale $(<100 \mathrm{~nm})$, smooth and uniform surface, and densely packed thin-film MOF particles to ensure fast transport of charge carriers across the thin film ${ }^{21}$. Furthermore, immobilization of nanocatalyst into the pores is needed to optimize the catalytic performance of C-MOF thin films. Currently, the main techniques to generate nanoscale thin films of C-MOFs are layer-by-layer ${ }^{14,22}$, and interfacial synthesis $6,23,24$. Although high-quality C-MOF films can be generated with these techniques, simultaneous immobilization of nanocatalyst during CMOF thin-film synthesis is difficult. In general, embedding of nanocatalyst within MOF films has been done by the following methods; ${ }^{25,26}$ (1) synthesis of metal nanoparticles (NPs) in (or on) pre-synthesized MOFs, (2) assembly of MOFs around presynthesized metal NPs, and (3) mixing all precursors and simultaneous growth of MOFs and metal NPs (in situ growth). The nanocatalyst-embedded MOF crystals are then deposited as a thin film ${ }^{11,12,27}$. However, these techniques inevitably require multiple relatively slow processes and often result in poor thinfilm quality. To the best of our knowledge, there is currently no technique to generate high-quality nanoscale C-MOF thin-film with embedded nanocatalyst particles within the pores.

Herein, we introduce microfluidic channel-embedded solution shearing (MiCS) as a means to immobilize metal NPs (i.e., nanocatalysts) into the C-MOFs pores during thin-film synthesis, through which high-quality nanocatalyst-embedded C-MOF thin films are generated with thickness control down to tens of nanometers. Furthermore, contrary to other MOF thin-film growth techniques ${ }^{14,22}$, MiCS can generate MOF thin film over a large area in a scalable and high-throughput $(5 \mathrm{~mm} / \mathrm{s})$ manner, rendering it a feasible technique for large-scale manufacturing. Solution shearing is a technique analogous to blade coating, where a fixed amount of solution is sandwiched between a moving blade and a heated substrate ${ }^{28-30}$. A meniscus (curved air-liquid interface) naturally forms between the blade and the substrate, and as the meniscus moves along with the moving blade, a thin film is deposited across the substrate as solution-tosolid transition occurs near the edge of the meniscus ${ }^{28}$.

\section{Results}

Preparation of nanocatalyst-embedded C-MOF thin films using MiCS. Figure $1 \mathrm{a}$ is a schematic depiction of metal NPembedded C-MOF thin-film formation using MiCS. Contrary to conventional solution shearing, in MiCS, microfluidic channels have been embedded within the blade, and these channels act as

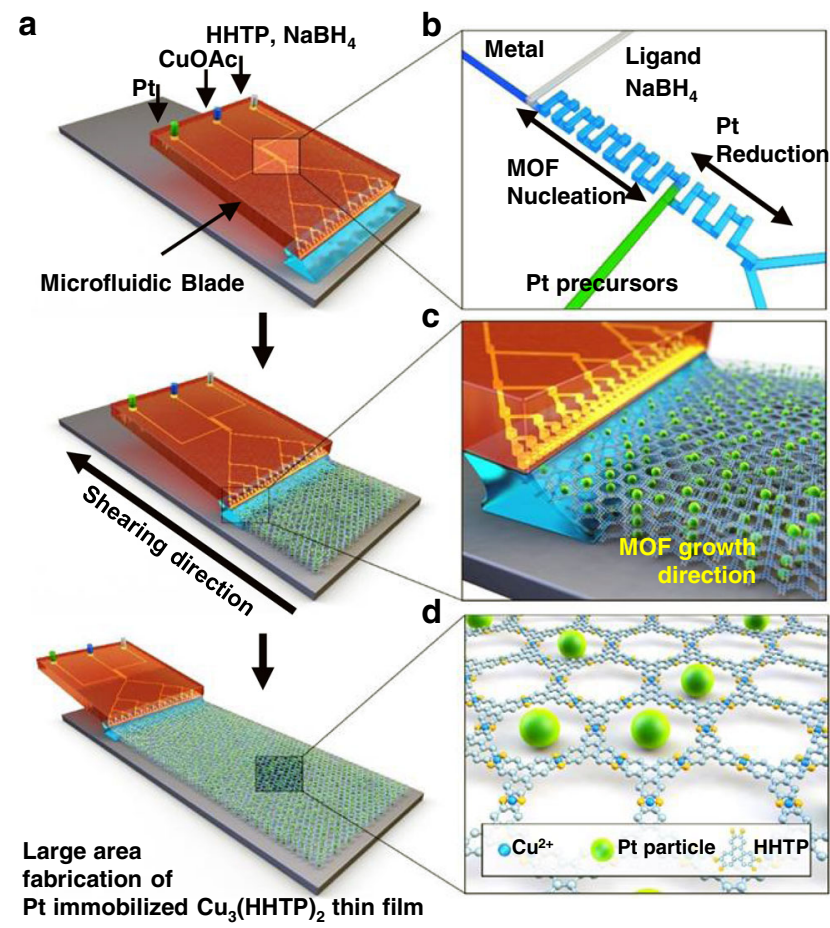

Fig. 1 Fabrication of $\mathbf{P t} @ \mathrm{Cu}_{\mathbf{3}}(\mathrm{HHTP})_{2}$ thin film using MiCS. a Schematic of Pt@Cu $u_{3}(\mathrm{HHTP})_{2}$ MOF thin-film processing using microfluidic-based solution-shearing process. CuOAC is copper(II) acetate. b Schematic illustration of the microfluidic blade channel. c Schematic illustration of the MOF growth process using solution shearing. The nucleated Pt particleembedded MOF solution is located between the microfluidic blade and the heated substrate. The MOF growth occurs at the edge of the meniscus. With the continuously supplying solution by the microfluidic blade during solution shearing, large-area and uniform Pt@ $\mathrm{Cu}_{3}(\mathrm{HHTP})_{2}$ thin film can be formed. d Crystal structure of Pt@ $\mathrm{Cu}_{3}(\mathrm{HHTP})_{2}$ thin film (green sphere: Pt particle). @ means -embedded.

microreactors for the chemical synthesis of nanocatalystembedded C-MOFs. As one of the widely used typical C-MOFs, we chose $\mathrm{Cu}_{3}$ (hexahydroxytriphenylene $)_{2}\left[\mathrm{Cu}_{3}(\mathrm{HHTP})_{2}\right]$ that has $2 \mathrm{D}$ structures with numerous rigid pores ${ }^{3} \cdot \mathrm{Cu}_{3}(\mathrm{HHTP})_{2}$ is generally synthesized by hydrothermal or solvothermal methods which take more than a few hours, and it has high utility in chemical sensors, supercapacitors, and other electronic devices $^{1-3,5}$. Figure $1 \mathrm{~b}$ is the depiction of the microfluidic channel design. $\mathrm{Cu}$ metal precursor solution and organic ligand $\left(2,3,6,7,10,11\right.$-hexahydroxytriphenylene)/reducing agent $\left(\mathrm{NaBH}_{4}\right)$ solution was firstly mixed together, inducing nucleation of $\mathrm{Cu}_{3}(\mathrm{HHTP})_{2}$ C-MOF particles. It is noted that $\mathrm{NaBH}_{4}$ plays a role in reducing Pt precursors at the next step. This solution was then mixed with Pt precursor solution, which immobilizes the $\mathrm{Pt}$ nanocatalyst particles within the $\mathrm{C}-\mathrm{MOF}$ pores. As the solution containing the Pt-embedded $\mathrm{Cu}_{3}(\mathrm{HHTP})_{2}\left(\mathrm{Pt} @ \mathrm{Cu}_{3}(\mathrm{HHTP})_{2}\right)$ particles flow out onto the heated substrate $\left(150^{\circ} \mathrm{C}\right)$, particles grow into a thin film on the substrate, as depicted in Fig. 1c and d. The gap (i.e., distance between microfluidic blade and substrate), angle, and temperature were set at $100 \mu \mathrm{m}, 30^{\circ}$, and $150^{\circ} \mathrm{C}$, which was the optimal condition to fabricate the Pt@ $\mathrm{Cu}_{3}(\mathrm{HHTP})_{2}$ thinfilm (Supplementary Figs. 1-3). In other words, our method induces (1) the ultrafast synthesis of C-MOFs and the reduction of metal NPs in microfluidic channels, and (2) the direct growth of metal NP-loaded C-MOFs on substrates by the solution shearing. Supplementary Table 1 summarizes the pros and cons of various MOF thin-film formation techniques. For fabrication 


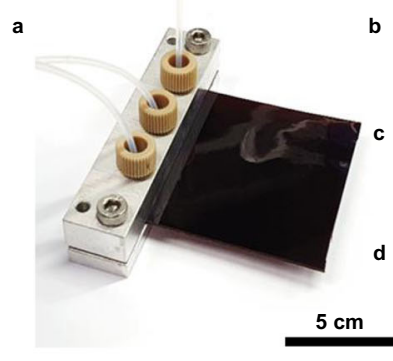

$1^{\text {st }}$ cycle

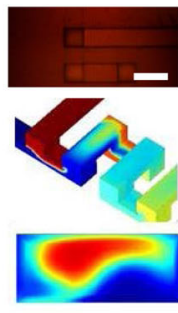

$9^{\text {th }}$ cycle

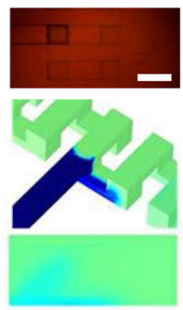

$15^{\text {th }}$ cycle

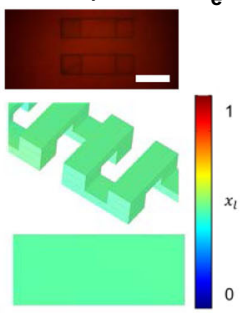

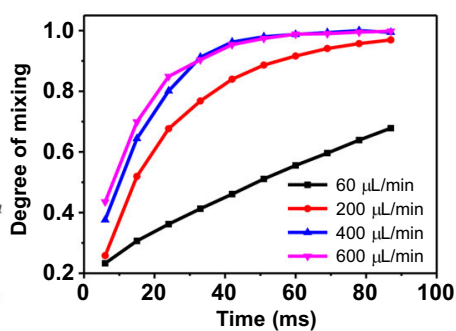
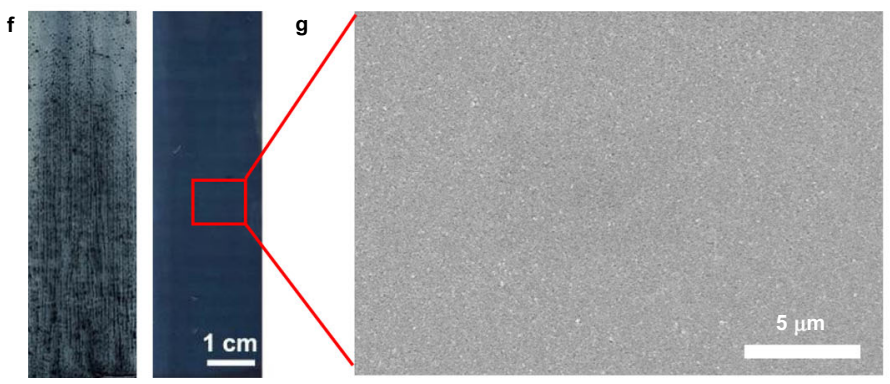

h

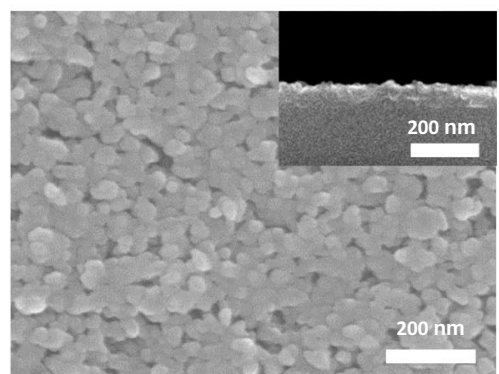

Fig. 2 Characterization of MiCS. a Photographic image of the polyimide microfluidic blade. b Optical image of polyimide microfluidic device channel (scale bar: $500 \mathrm{~mm}$ ): 1st cycle (left), 9th cycles (middle), 15th cycles (right). c 3D surface and d 2D cross-sectional concentration distribution of solution ( $x_{l}$ ) at 1st (left), 9th (middle), and 15th (right) cycle under $660 \mu \mathrm{L} / \mathrm{min}$ flow rate. e Effect of cycle number on the degree of mixing obtained at various flow rates. f Optical image of solution sheared Pt@Cu $(\mathrm{HHTP})_{2}$ film (shearing speed at $2 \mathrm{~mm} / \mathrm{s}$ ) on glass substrate at $150{ }^{\circ} \mathrm{C}$ : without microfluidic blade (left), and with microfluidic blade (right).g, SEM image of Pt@ $\mathrm{Cu}_{3}(\mathrm{HHTP})_{2}$ thin film. h High-resolution SEM image of Pt@Cu $3(\mathrm{HHTP})_{2}$ thin film. @ means -embedded.

of metal NP-embedded MOF thin films, conventional methods involve the synthesis of MOFs, the pre- or post-introduction of metal NPs (or in situ synthesis of metal NPs and MOFs), and the post process for film fabrication. However, MiCS is a unique technique where the synthesis of C-MOFs, decoration of nanocatalyst, and the growth of thin film occurs concurrently all in a single step. Therefore, our method enables the ultrafast and largearea synthesis of metal NP-embedded C-MOF thin films. In order for MiCS to work properly, the synthesis of $\mathrm{Pt} @ \mathrm{Cu}_{3}(\mathrm{HHTP})_{2}$ particles must occur on the same time scale as that of the solution shearing rate. In this regard, the use of microfluidic reactors enable fast mixing and mass transfer effects unachievable by batch systems, leading to significantly enhanced reaction rates $^{31-33}$. Hence, despite the short reaction time, continuous synthesis of $\mathrm{Pt} @ \mathrm{Cu}_{3}(\mathrm{HHTP})_{2}$ particles during solution shearing is possible. Furthermore, the fast reaction rate and precisely controlled reaction time yield uniformly sized Pt NPs and $\mathrm{Cu}_{3}(\mathrm{HHTP})_{2}$ particles.

Figure $2 \mathrm{a}$ is an optical image of the microfluidic channelembedded solution-shearing blade; further details regarding the microfluidic chip design are described in Supplementary Fig. 4. The microfluidic channels were designed as a three-dimensional serpentine mixer (Supplementary Fig. 5) due to its high mixing efficiency ${ }^{34}$. Figure $2 b$ shows top view optical images of the microfluidic channels at various cycling points, and Fig. $2 \mathrm{c}$ and $\mathrm{d}$ shows the corresponding computational fluid dynamics (CFD) simulation results depicting the degree of mixing. As the number of cycles increases, the degree of mixing increases, as indicated by the appearance of the light-green-colored solution. The flow rate was optimized using CFD simulation, where various flow rates were evaluated by monitoring the degree of mixing as a function of the number of cycles (Supplementary Tables 2-5). When the flow rate exceeded $220 \mu \mathrm{L} / \mathrm{min}, 15$ mixing cycles were sufficient to fully mix the solutions, as seen in Fig. 2e. At this flow rate, the solution residence time within the microfluidic channel is short (200 ms, Supplementary Fig. 6), which prevented the microfluidic channels from being clogged due to particle aggregation. Figure $2 \mathrm{f}$ shows optical images of the Pt@ $\mathrm{Cu}_{3}(\mathrm{HHTP})_{2}$ thin film using conventional solution shearing (left) and MiCS (right). In the case of solution shearing, all of the components were premixed as a bulk solution, and the solution was inserted in between the blade and the substrate prior to coating. Over a large area, the thin film coated with MiCS exhibited a high degree of uniformity without the presence of particle aggregates. This was on the contrary to thin film coated with conventional solution shearing, which visually showed variability in film color and particle aggregates. The surface roughness of the film made with MiCS was $2.8 \mathrm{~nm}$, whereas that of the solution shearing was $92.3 \mathrm{~nm}$ (Supplementary Fig. 7). Such thin-film properties attained using MiCS can be attributed to the controlled nucleation reaction of the precursor solution within the microfluidic channel, and the continuous supply of solution that maintains constant solution volume, concentration, and the shape of the meniscus during coating. These factors can alter the fluid behavior near the meniscus, which can change the thin-film properties ${ }^{35}$.

Characterizations of Pt@C $\mathbf{C u}_{3}(\mathbf{H H T P})_{2}$ thin film. Figure $2 \mathrm{~g}$ and $\mathrm{h}$ shows scanning electronic microscopy (SEM) images of the $\mathrm{Pt} @ \mathrm{Cu}_{3}(\mathrm{HHTP})_{2}$ thin film fabricated using MiCS, confirming the closely packed MOF thin film. The average crystallite size of $\mathrm{Pt} @ \mathrm{Cu}_{3}(\mathrm{HHTP})_{2}$ in the thin film is $25 \mathrm{~nm}$ (Supplementary Fig. 8). Powder X-ray diffraction (PXRD) was conducted on $\mathrm{Pt} @ \mathrm{Cu}_{3}(\mathrm{HHTP})_{2}$ thin film grown on the substrate and on the nucleated Pt@ $\mathrm{Cu}_{3}(\mathrm{HHTP})_{2}$ particles being extruded out of the microfluidic channels (without undergoing thin film growth on the substrate) (Supplementary Fig. 9). Much weaker diffraction peaks were observed for the latter case, which confirms that $\mathrm{Pt} @ \mathrm{Cu}_{3}(\mathrm{HHTP})_{2}$ thin-film is grown on the substrate after being extruded out of the microfluidic channels. Previous C-MOF growth techniques indicate that elevated temperature is needed for thin-film growth since energy is required to form coordination bonds; this corroborates the XRD results ${ }^{36}$. As stated above, the simultaneous synthesis and growth of thin film is a unique 

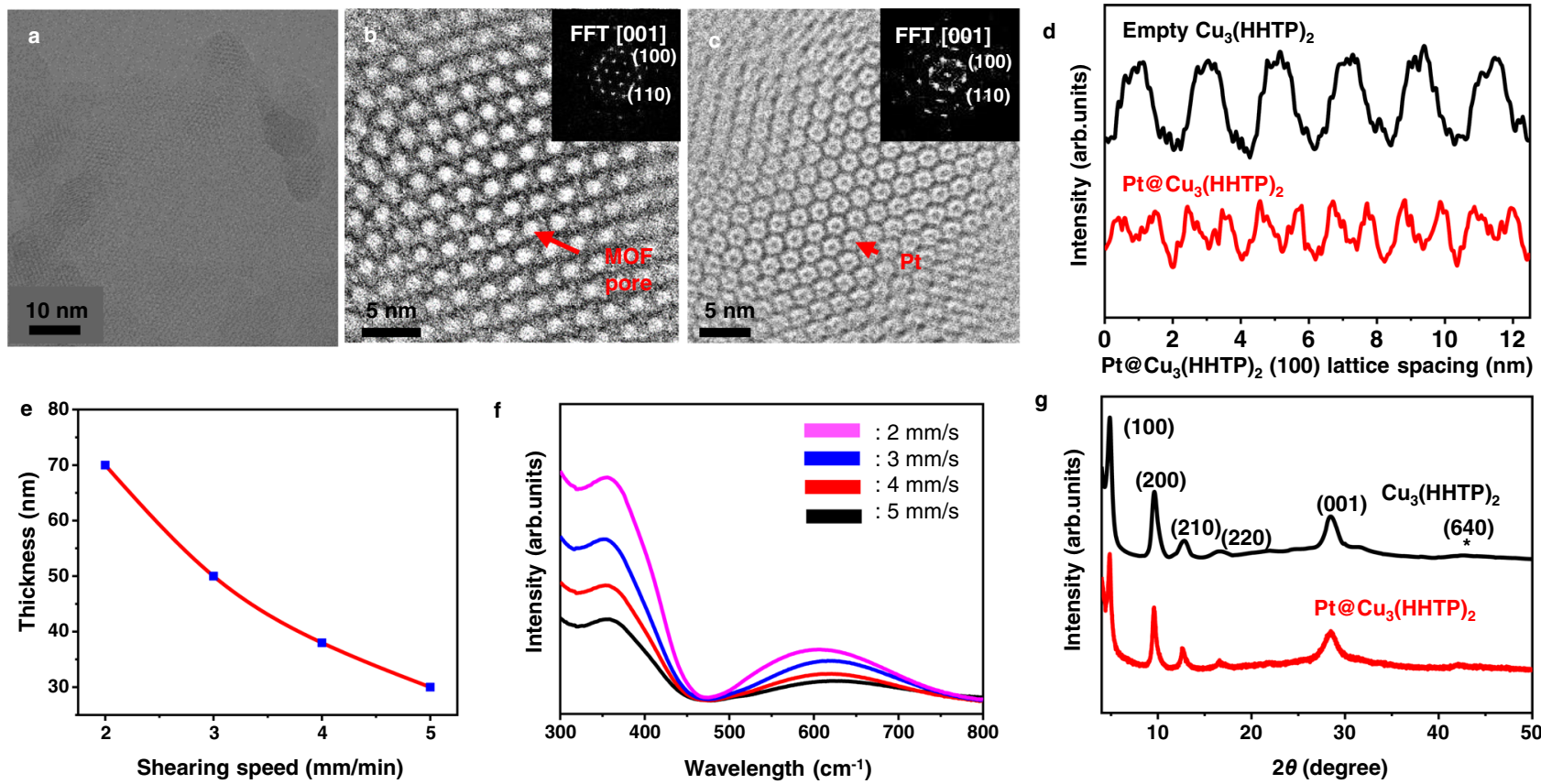

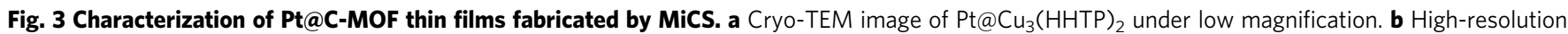
Cryo-TEM image of Pt@Cu $\mathrm{Cu}_{3}(\mathrm{HHTP})_{2}$ without Pt particles in the MOF pores. c High-resolution Cryo-TEM image of Pt particles immobilized in the MOF pores. d Integrated intensity of Pt@ $\mathrm{Cu}_{3}(\mathrm{HHTP})_{2}$ plotted over six unit cells along the [100] direction, indicating a pattern when Pt particle is introduced into the pores. e Thickness control of Pt@Cu $(\mathrm{HHTP})_{2}$ thin film via controlling the shearing speeds. $\mathbf{f} \mathrm{UV}$-Vis spectrum of different thicknesses of

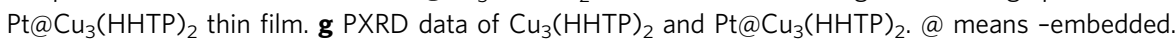

feature of MiCS, which allows the formation of a densely packed high-quality film with low surface roughness and nanoscale thickness control (discussed below).

To confirm the immobilization of Pt NPs within the MOF pores, cryo-transmission electronic microscopy (Cyro-TEM) was used to attain (001) plane view images of Pt@ $\mathrm{Cu}_{3}(\mathrm{HHTP})_{2}$, as depicted in Fig. $3 a-c$. Figure $3 b$ and $c$ is a close-up of the MOF pores in the region absent of and immobilized with Pt particles, respectively, showing a clear difference in the image contrast. Figure $3 \mathrm{~d}$ plots the integrated pixel intensities for the empty $\mathrm{Cu}_{3}(\mathrm{HHTP})_{2}$ and Pt immobilized $\mathrm{Cu}_{3}(\mathrm{HHTP})_{2}$ unit cells along the [100] direction. The [100] lattice spacing $\left(\mathrm{d}_{100}\right)$ for the empty $\mathrm{Cu}_{3}(\mathrm{HHTP})_{2}$ across five unit cells was measured to be c.a. $2.0 \mathrm{~nm}$, which is in good agreement with the theoretical pore size of $\mathrm{Cu}_{3}(\mathrm{HHTP})_{2}{ }^{22}$. In the case of Pt@Cu $\mathrm{Cu}_{3}(\mathrm{HHTP})_{2}$, since the beam cannot pass through the $\mathrm{Pt}$ particles, darker regions within the pores were observed. To further identify the Pt particles, highresolution-TEM (HRTEM) was used to damage the MOF structure and observe the Pt particles, as depicted in Supplementary Fig. 10. As shown in Supplementary Fig. 10a, Pt particles having an average diameter of $2 \mathrm{~nm}$ are well-dispersed in $\mathrm{Cu}_{3}(\mathrm{HHTP})_{2}$. Supplementary Fig. 10b shows the lattice of the Pt (111) plane with a spacing of $2.265 \AA$. Scanning TEM (STEM) image of Pt@ $\mathrm{Cu}_{3}(\mathrm{HHTP})_{2}$ demonstrated that the Pt particles are well-dispersed throughout the $\mathrm{Cu}_{3}(\mathrm{HHTP})_{2}$ structure (Supplementary Fig. 10c). TEM-EDS element mapping was used to confirm the presence of $\mathrm{Cu}, \mathrm{C}, \mathrm{O}$, and $\mathrm{Pt}$ in the sample (Supplementary Fig. 10d). Comparing the HRTEM images of $\mathrm{Pt} @ \mathrm{Cu}_{3}(\mathrm{HHTP})_{2}$ made using conventional bulk synthesis technique $^{15}$ to that of MiCS (Supplementary Fig. 11) showed that the size variance of the Pt particles was much higher for the former. This can be attributed to the rapid and controlled $\mathrm{Pt}$ reduction reaction within the microfluidic channels ${ }^{37}$.

The thickness of Pt@ $\mathrm{Cu}_{3}(\mathrm{HHTP})_{2}$ thin film can be precisely controlled at tens of nanometer level using MiCS (Fig. 3e). The film thickness at various shearing speeds was confirmed using atomic force microscopy (AFM) images (Supplementary Fig. 12). The thickness decreasing with increasing shearing speed indicates that the system is in an evaporative regime, where the solvent evaporation rate is on a similar time scale to the shearing rate ${ }^{38}$. In this regime, thickness variation can theoretically be explained by the mass balance between the evaporating solvent and the depositing thin film to the solution flowing towards the meniscus ${ }^{38}$, with a power law, thickness $\propto$ speed $^{-1}$. Our data indicate a power dependence of -0.99 , which is in good agreement with the theoretical value. Ultraviolet-visible (UV/

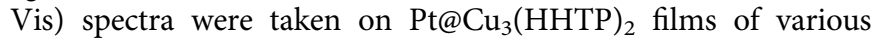
thicknesses, as seen in Fig. 3f. The characteristic absorbance at $363 \mathrm{~nm}\left(\pi-\pi^{*}\right.$ transition) and $645 \mathrm{~nm}$ (ligand to metal charge transfer band) increased with decreasing the shearing speed due to the increasing film thickness ${ }^{14,22}$. The crystal structures of $\mathrm{Cu}_{3}(\mathrm{HHTP})_{2}$ and $\mathrm{Pt} @ \mathrm{Cu}_{3}(\mathrm{HHTP})_{2}$ thin films were investigated by powder X-ray diffraction (PXRD) analysis (Fig. 3g). The pristine $\mathrm{Cu}_{3}(\mathrm{HHTP})_{2}$ showed the crystal planes of (100), (200), (210), (220), and (001), which was similar to the observation in

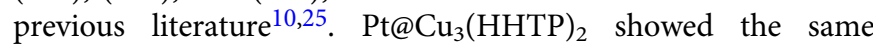
diffraction pattern as pristine $\mathrm{Cu}_{3}(\mathrm{HHTP})_{2}$ without a clear appearance of $\mathrm{Pt}$ diffraction peak (at $2 \theta=42^{\circ}$ ). It is noted that the XRD result of bulk-grown Pt@ $\mathrm{Cu}_{3}(\mathrm{HHTP})_{2}$ powders also displayed similar patterns with those of Pt@ $\mathrm{Cu}_{3}(\mathrm{HHTP})_{2}$ thin films (Supplementary Fig. 13), revealing that $\mathrm{NaBH}_{4}$ likely does not affect the crystal structure of C-MOF. This can be attributed to the very small amount of Pt particles and their embedment into the MOF pores ${ }^{19}$. The $\mathrm{N}_{2}$ adsorption and desorption isotherms at $77 \mathrm{~K}$ confirmed the porous nature of $\mathrm{Cu}_{3}(\mathrm{HHTP})_{2}$ and Pt@ $\mathrm{Cu}_{3}(\mathrm{HHTP})_{2}$ (Supplementary Fig. 14). The Brunauer-Emmett-Teller (BET) surface area of Pt@ $\mathrm{Cu}_{3}(\mathrm{HHTP})_{2}$ was $200 \mathrm{~m}^{2} \mathrm{~g}^{-1}$, which showed a decreased value than the surface area of $\mathrm{Cu}_{3}(\mathrm{HHTP})_{2}\left(250 \mathrm{~m}^{2} \mathrm{~g}^{-1}\right)$. This can be ascribed to the $\mathrm{Pt}$ particles blocking the MOF pores ${ }^{11}$. 
Sensing characteristics of $\mathrm{Pt} @ \mathrm{Cu}_{3}(\mathrm{HHTP})_{2}$ thin film. To demonstrate the ultrahigh catalytic activity and potential applications of $\mathrm{Pt} @ \mathrm{Cu}_{3}(\mathrm{HHTP})_{2}$ thin film, we investigated its chemiresistive sensing properties. Chemiresistors are in high demand for a wide range of applications, such as environmental monitoring 39 , exhaled breath analysis ${ }^{40}$, and food quality control ${ }^{41}$. However, there are grand challenges in chemiresistive sensors: low responses to sub-ppm levels of analytes, poor crossselectivity, and high-power consumption. We postulated that our thin films can address these issues because they have high surface area and porosity, ultrahigh reactivity, and can be operated at room temperature. In addition, since MiCS-based thin film has uniform electrical characteristics (see Supplementary Fig. 15 for comparison between MiCS-based and conventional solutionshearing-based thin-film electrical properties), relatively reliable sensing data can be attained. We fabricated chemiresistors using $\mathrm{Pt} @ \mathrm{Cu}_{3}(\mathrm{HHTP})_{2}$ thin film along with control samples $\left(\mathrm{Cu}_{3}(\mathrm{HHTP})_{2}\right.$ powder and pristine $\mathrm{Cu}_{3}(\mathrm{HHTP})_{2}$ thin film) (see details in the "Methods"). First, to optimize the $\mathrm{NO}_{2}$ sensing properties of $\mathrm{Pt} @ \mathrm{Cu}_{3}(\mathrm{HHTP})_{2}$ thin-film-based sensors at room temperature in air, we controlled the loading amounts of Pt NPs by the different flow rates of Pt precursors (50, 100, 150, and 200 $\mu \mathrm{L} / \mathrm{min}$ ) (Supplementary Fig. 16). The weight ratio of Pt-loaded $\mathrm{Cu}_{3}(\mathrm{HHTP})_{2}$ was calculated using Inductively Coupled Plasma Mass Spectrometer analysis (ICP-MS) (Supplementary Table 6). Among the samples, the $2.3 \mathrm{wt} \%$ Pt-loaded $\mathrm{Cu}_{3}(\mathrm{HHTP})_{2}$ thin film exhibited the optimum $\mathrm{NO}_{2}$ sensing properties (hereafter, the $2.3 \mathrm{wt} \%$ Pt-loaded $\mathrm{Cu}_{3}(\mathrm{HHTP})_{2}$ thin film represents the $\mathrm{Pt} @ \mathrm{Cu}_{3}(\mathrm{HHTP})_{2}$ thin-film-based $\mathrm{NO}_{2}$ sensors).

The Pt@ $\mathrm{Cu}_{3}(\mathrm{HHTP})_{2}$ thin film displays significant resistance changes $\left(\Delta \mathrm{R} / \mathrm{R}_{\mathrm{a}}=-89.9 \%\right)$ at 3 ppm of $\mathrm{NO}_{2}$ (Fig. $\left.4 \mathrm{a}\right)$. In contrast, the $\mathrm{Cu}_{3}(\mathrm{HHTP})_{2}$ powder and $\mathrm{Cu}_{3}(\mathrm{HHTP})_{2}$ thin film show lower responses $\left(-11.8 \%\right.$ for $\mathrm{Cu}_{3}(\mathrm{HHTP})_{2}$ powder and $-53.7 \%$ for $\mathrm{Cu}_{3}(\mathrm{HHTP})_{2}$ thin film) relative to the Pt@ $\mathrm{Cu}_{3}(\mathrm{HHTP})_{2}$ thin film. In addition, the $\mathrm{Pt} @ \mathrm{Cu}_{3}(\mathrm{HHTP})_{2}$ and $\mathrm{Cu}_{3}(\mathrm{HHTP})_{2}$ thin films are able to detect $\mathrm{NO}_{2}$ as low as $0.1 \mathrm{ppm}$, whereas the $\mathrm{Cu}_{3}(\mathrm{HHTP})_{2}$
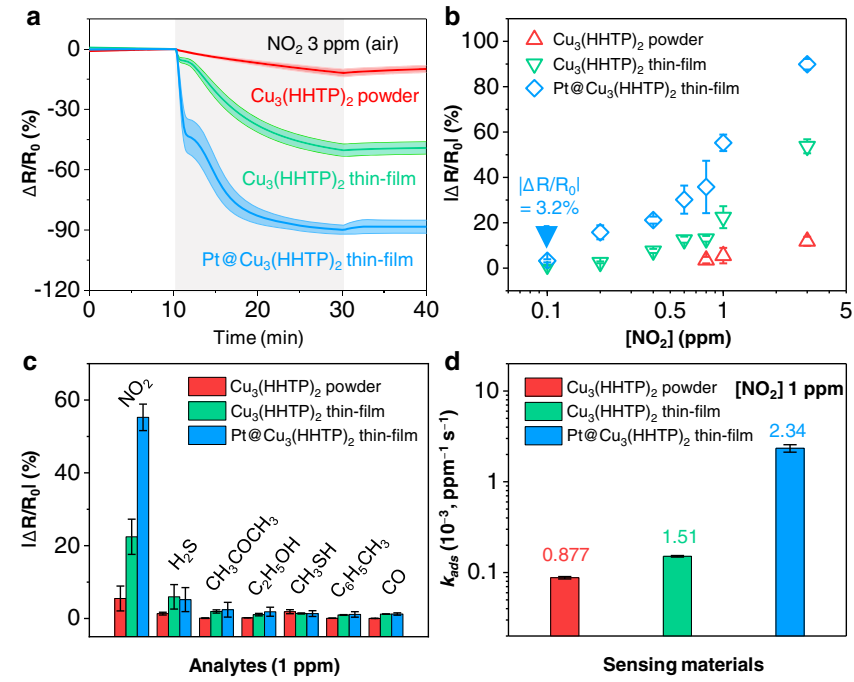

Fig. 4 Sensing characteristics of $\mathrm{Pt} @ \mathrm{Cu}_{3}(\mathrm{HHTP})_{2}$ thin film (shearing speed: $2 \mathrm{~mm} / \mathrm{s}$ ) on alumina $\left(\mathrm{Al}_{2} \mathrm{O}_{3}\right)$ substrate at $150{ }^{\circ} \mathrm{C}$. a Resistance changes of $\mathrm{Cu}_{3}(\mathrm{HHTP})_{2}$ powder, $\mathrm{Cu}_{3}(\mathrm{HHTP})_{2}$ thin film, and

$\mathrm{Pt} @ \mathrm{Cu}_{3}(\mathrm{HHTP})_{2}$ thin film in response to 20 min exposure of $\mathrm{NO}_{2} 3 \mathrm{ppm}$. The shaded areas present the standard deviation of the sensors $(N \geq 4)$. $\mathbf{b}$ Normalized responses of the sensors to $0.1-3 \mathrm{ppm}$ of $\mathrm{NO}_{2}$. c Selectivity of the sensors to $1 \mathrm{ppm}$ of analytes. $\mathbf{d}$ Calculated adsorption rate constants $\left(k_{\text {ads }}\right)$ of the sensors. @ means -embedded. The error bars in $\mathbf{b}-\mathbf{d}$ show the standard deviation of the sensors $(N \geq 4)$. powder do not function below 1 ppm (Supplementary Fig. 17). We calculated normalized responses $\left(\left|\Delta \mathrm{R} / \mathrm{R}_{0}\right|\right)$ and response times $\left(t_{90}\right)$ of the sensors in the range of $\mathrm{NO}_{2} 0.1-3$ ppm (Fig. $4 \mathrm{~b}$ and Supplementary Fig. 18). The formation of thin-film structure $\left(\mathrm{Cu}_{3}(\mathrm{HHTP})_{2}\right.$ thin film $)$ improves the responses of $\mathrm{Cu}_{3}(\mathrm{HHTP})_{2}$ by a factor of 4 , compared to $\mathrm{Cu}_{3}(\mathrm{HHTP})_{2}$ powder. In addition to the structural effect, the decoration of Pt NPs $\left(\mathrm{Pt} @ \mathrm{Cu}_{3}(\mathrm{HHTP})_{2}\right.$ thin film) induces twofold higher responses than pristine $\mathrm{Cu}_{3}(\mathrm{HHTP})_{2}$ thin-film. Furthermore, the Pt@Cu $\mathrm{Cu}_{3}(\mathrm{HHTP})_{2}$ thinfilm shows faster-sensing speed (8.2 min to $\left.\mathrm{NO}_{2} 3 \mathrm{ppm}\right)$ compared to the $\mathrm{Cu}_{3}(\mathrm{HHTP})_{2}$ thin-film $(14 \mathrm{~min})$ and $\mathrm{Cu}_{3}(\mathrm{HHTP})_{2}$ powder $(17.3 \mathrm{~min})$. These results demonstrated that both the thin-film structure of $\mathrm{Cu}_{3}(\mathrm{HHTP})_{2}$ and ultra-small Pt NPs increase the activity of the sensors. Although the response times of the $\mathrm{Pt} @ \mathrm{Cu}_{3}(\mathrm{HHTP})_{2}$ thin film are sluggish at low $\mathrm{NO}_{2}$ concentrations $(<1 \mathrm{ppm})$, the Pt@ $\mathrm{Cu}_{3}(\mathrm{HHTP})_{2}$ thin-film-based sensors (13 min for $\mathrm{NO}_{2} 1 \mathrm{ppm}$ ) meet the safety standard for $\mathrm{NO}_{2}$ exposures; the short-term permissible exposure limit of $\mathrm{NO}_{2}$, designated by the Occupational Safety and Health Administration (OSHA) in the USA, is $1 \mathrm{ppm}$ within $15 \mathrm{~min}$.

We further investigated the other important parameters for $\mathrm{NO}_{2}$ sensors: selectivity and stability. The Pt@Cu $3(\mathrm{HHTP})_{2}$ thinfilm sensors display ultrahigh $\mathrm{NO}_{2}$ cross-selectivity against other interfering analytes (hydrogen sulfide $\left[\mathrm{H}_{2} \mathrm{~S}\right]$, acetone $\left[\mathrm{CH}_{3} \mathrm{COCH}_{3}\right]$, ethanol $\left[\mathrm{C}_{2} \mathrm{H}_{5} \mathrm{OH}\right]$, methanethiol $\left[\mathrm{CH}_{3} \mathrm{SH}\right]$, toluene $\left[\mathrm{C}_{6} \mathrm{H}_{5} \mathrm{CH}_{3}\right]$, and carbon monoxide [CO]) (Fig. 4c). The responses (55.3\%) of the $\mathrm{Pt} @ \mathrm{Cu}_{3}(\mathrm{HHTP})_{2}$ thin film to $\mathrm{NO}_{2}$ are tenfold higher than those $(>5.2 \%)$ to other gas molecules. In addition, the sensors show stable responses to multiple exposures to $\mathrm{NO}_{2} 0.1 \mathrm{ppm}$ (Supplementary Fig. 19a), demonstrating high feasibility as dosimetric sensors. Furthermore, the $\mathrm{Pt} @ \mathrm{Cu}_{3}(\mathrm{HHTP})_{2}$ thin-film-based sensors function well in humid air (relative humidity $=95 \%$ ) (Supplementary Fig. 19b). There are slight decreases in the response $\left(-38.9 \%\right.$ to $\left.\mathrm{NO}_{2} 1 \mathrm{ppm}\right)$ and response speed (18 $\mathrm{min}$ ) under the humid air (relative humidity: 90\%) compared to those (response $=-55.3 \%$ and response time $=13 \mathrm{~min}$ ) under the dry air (relative humidity: 5\%). These decreases are attributed to the fact that adsorbed water molecules retard the reactions of $\mathrm{NO}_{2}$ on $\mathrm{Pt} @ \mathrm{Cu}_{3} \mathrm{HHTP}_{2}$ thin film. Nonetheless, these sensing performances demonstrate that our sensors represent a viable $\mathrm{NO}_{2}$ sensor operated at room temperature in air.

To elucidate the structural and catalytic effect of Pt@ $\mathrm{Cu}_{3}(\mathrm{HHTP})_{2}$ thin film on $\mathrm{NO}_{2}$ sensing, we calculated adsorption rate kinetics $\left(k_{\mathrm{ads}}\right)$ using response traces of the sensors. Since the origin of chemiresistive sensing of $\mathrm{Cu}_{3}(\mathrm{HHTP})_{2}$ is charge transfers caused by adsorbed gas molecules ${ }^{12,42}$, with the assumption that the responses of $\mathrm{Cu}_{3}(\mathrm{HHTP})_{2}$ are proportional to the amounts of adsorbed $\mathrm{NO}_{2}$ molecules, reaction rate constants are obtained by fitting response traces to theoretical equations (see details in Supplementary Fig. 20 and Supplementary Table 7). The calculated $k_{\text {ads }}$ values are described in Fig. $4 \mathrm{~d}$. The Pt@Cu $\mathrm{Cu}_{3}(\mathrm{HHTP})_{2}$ thin film shows higher $\mathrm{NO}_{2}$ adsorption kinetics $\left(k_{\mathrm{ads}}=2.34 \times 10^{-3} \mathrm{ppm}^{-1} \mathrm{~s}^{-1}\right)$ than the $\mathrm{Cu}_{3}(\mathrm{HHTP})_{2}$ powder $\left(0.877 \times 10^{-3} \mathrm{ppm}^{-1} \mathrm{~s}^{-1}\right)$ and the $\mathrm{Cu}_{3}(\mathrm{HHTP})_{2}$ thin film $\left(1.51 \times 10^{-3} \mathrm{ppm}^{-1} \mathrm{~s}^{-1}\right)$, demonstrating that $\mathrm{NO}_{2}$ reactions on $\mathrm{Cu}_{3}(\mathrm{HHTP})_{2}$ are promoted by two factors: (1) the thin-film structure of $\mathrm{Cu}_{3}(\mathrm{HHTP})_{2}$ and (2) the catalytic effect of ultra-small Pt NPs. The thin-film structure induces high gas accessibility into sensing layers ${ }^{14}$, and ultra-small Pt NPs $(\sim 2 \mathrm{~nm})$ cause $\mathrm{NO}_{2}$ spillover onto $\mathrm{Cu}_{3}(\mathrm{HHTP})_{2}{ }^{15}$. Therefore, $\mathrm{NO}_{2}$ molecules are easily accessible to $\mathrm{Cu}_{3}(\mathrm{HHTP})_{2}$ layers, and their reactions are activated by nanoscopic Pt catalysts, leading to high sensing performances. These sensing performances are higher than other 2D material-based $\mathrm{NO}_{2}$ chemiresistors operated at room temperature in air (Fig. 5 and Supplementary Table 8) 8, $^{15-52}$. 


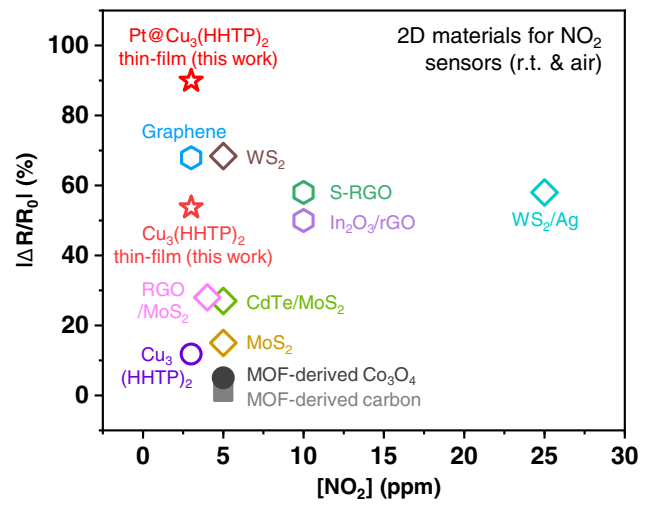

Fig. 5 Comparison of the $\mathrm{NO}_{2}$ responses of $\mathrm{Pt} @ \mathrm{Cu}_{3}(\mathrm{HHTP})_{2}$ thin-film and $\mathrm{Cu}_{\mathbf{3}}$ (HHTP) $)_{2}$ thin-film with those of other materials. Other materials include $\mathrm{Cu}_{3}(\mathrm{HHTP})_{2}$, graphene, $\mathrm{WS}_{2}, \mathrm{RGO} / \mathrm{MoS}_{2}$ (RGO: reduced graphene oxide), $\mathrm{CdTe} / \mathrm{MoS}_{2}, \mathrm{MoS}_{2}, \mathrm{MOF}$-derived $\mathrm{CO}_{3} \mathrm{O}_{4}, \mathrm{MOF}$-derived carbon, S$\mathrm{RGO}$ (S-RGO: sulfonated RGO), $\ln _{2} \mathrm{O}_{3} / \mathrm{RGO}$, and $\mathrm{WS}_{2} / \mathrm{Ag}$. / means heterostructured with.

In particular, the $\mathrm{NO}_{2}$ responses of our sensors are hugely improved compared to MOF-based $\mathrm{NO}_{2}$ sensors.

\section{Discussion}

C-MOFs are emerging materials with a high degree of chemical versatility and ultrahigh porosity, rendering them a suitable material for next-generation electronics, energy storage devices, and sensors. To fully take advantage of C-MOFs, the generation of high-quality nanoscale thin films with the versatility to immobilize nanocatalysts into the MOF pores is of critical importance. This has thus far been a major challenge as the simultaneous synthesis of MOF thin-film and immobilization of nanocatalysts is difficult. Furthermore, high-throughput, largearea, single-step (synthesis, film growth, catalyst immobilization) thin-film generation remains challenging, limiting the commercial feasibility of C-MOFs. Our proposed MiCS technique overcomes these difficult challenges. The use of microfluidic channels enables the synthesis of catalyst-embedded MOF particles, which simultaneously grow into high-quality nanoscale thin-film in a large-area scalable, high-throughput manner. Our thin films exhibit significantly improved gas sensing performance, as a consequence of (1) the high porosity and facile gas accessibility of C-MOF thin films, and (2) the ultrahigh catalytic activity of the embedded NPs (c.a. $2 \mathrm{~nm}$ ). These results together point to the exciting prospects of utilizing MiCS for the generation and optimization of a variety of catalyst-embedded C-MOF thin films for various applications in the future.

\section{Methods}

Materials. All chemicals and solvents were of reagent grade and were used as received without further purification. Copper(II) acetate monohydrate $(\mathrm{Cu}$ $\left(\mathrm{CO}_{2} \mathrm{CH}_{3}\right) \mathrm{H}_{2} \mathrm{O}, 99.9 \%$ ), dimethyl sulfoxide (DMSO), potassium tetrachloroplatinate(II) $\left(\mathrm{K}_{2} \mathrm{PtCl}_{4}\right)$, and sodium borohydride $\left(\mathrm{NaBH}_{4}, 96 \%\right)$ were purchased from Sigma-Aldrich. 2,3,6,7,10,11-hexahydroxytriphenylene hydrate (HHTP, $\mathrm{C}_{18} \mathrm{H}_{12} \mathrm{O}_{6} \mathrm{H}_{2} \mathrm{O}$, 95.0\%) was purchased from Tokyo Chemical Industry. Glass, silicon wafer, and alumina $\left(\mathrm{Al}_{2} \mathrm{O}_{3}\right)$ were used as a substrate, and all substrates were treated with oxygen plasma for 1 min prior to coating of MOF thinfilm.

Mathematical formulation and numerical details. The flow behavior and mixing progress in the microfluidic mixing region were investigated numerically based on the finite element method (FEM) with COMSOL Multiphysics software (v5.5, Comsol Inc.). The computation of steady-state fluid flow was followed by the mass transfer analysis based on the derived velocity profile.

The flow regime $(\operatorname{Re} \sim \mathcal{O}(10) \ll 2000)$ for this work was considered as laminar flow, so the turbulent model was not involved ${ }^{34}$. The velocity $(\mathbf{v})$ and pressure $(p)$ profile were derived by numerically solving the governing equations as below:

$$
\begin{gathered}
\rho(\mathbf{v} \cdot \nabla) \mathbf{v}=-\nabla \cdot[-p \mathbf{I}+\mathbf{K}]: \text { Navier }- \text { Stokes equation, } \\
\nabla \cdot \mathbf{v}=0 \text { :continuity equation, } \\
\mathbf{K}=\mu\left(\nabla \mathbf{v}+(\nabla \mathbf{v})^{\mathrm{T}}\right): \text { constitutive equation, }
\end{gathered}
$$

where the density $(\rho)$ and viscosity $(\mu)$ followed the solvent's properties, dimethyl sulfoxide (DMSO, whose $\rho=1.1 \mathrm{~g} / \mathrm{mL}$ and $\mu=2 \mathrm{mPa} \cdot \mathrm{s}$ ). Concentration dependence on viscosity and density could be neglected based on the comparison of calculation results under similar conditions in the previous work ${ }^{34}$. As boundary conditions, the mass flow rate for inlet, nonslip condition on the inner surface of the wall, and ambient air pressure on the outlet were applied.

The concentration change of $\mathrm{NaBH}_{4}$ ligand $\left(C_{\mathrm{l}}\right)$, CuOAc metal $\left(C_{\mathrm{m}}\right)$, and $\mathrm{Pt}$ precursors $\left(C_{\mathrm{Pt}}\right)$ in DMSO were derived by solving governing equations for mass transfer as below:

$$
\begin{gathered}
\nabla \cdot \mathbf{J}_{\mathrm{i}}+\mathbf{v} \cdot \nabla c_{\mathrm{i}}=R_{\mathrm{i}}: \text { mass conservation equation, } \\
\mathbf{J}_{\mathrm{i}}=-D \nabla c_{\mathrm{i}}: \text { Fick's diffusion equation, }
\end{gathered}
$$

where the generic molecular diffusivity $\left(10^{-9} \mathrm{~m}^{2} / \mathrm{s}\right)$ were applied for the molecular diffusivity $(D)$. Because the Péclet number for this study is in the range of $10^{3} \leq P e \leq 2 \times 10^{4}$, convection-mediated mass transfer dominates mass transfer. Nucleation and catalyst embedding are sufficiently fast to assume that the degree of mixing directly indicates the conversion of HTTP nucleation and Pt reduction. In other words, under steady-state conditions, the right-hand side of the mass conservation equation was considered zero. For the boundary condition of mass transfer, the individual initial concentrations for each inlet and no mass flux gradient for the outlet were applied.

The geometry exactly the same as the microfluidic blade was manually constructed and was divided into computational elements in COMSOL, where the number of elements was determined by the mesh size dependency test. To achieve adequate calculation accuracy within the limits allowed by computational power $(2.1 \mathrm{GHz} 24$ core CPU, $250 \mathrm{~GB}$ RAM), first, second, and quadratic order discretization was applied for pressure, velocity, and concentration, individually.

Microfluidic cycle and degree of mixing $(\varepsilon)$. For indication and evaluation, the entire microfluidic blade was divided into microfluidic cycles as defined in Supplementary Fig. 5. Except for the first cycle, each cycle includes four turns and experiences the same residence time determined by the mass flow rate. The plane between the $n$th cycle and $n+1$ th cycle is defined as $\mathrm{P}_{n+1}(n \geq 1)$, and $\mathrm{P}_{1}$ is considered as the plane where the ligand and metal first meet, so the first cycle has only two turns after $\mathrm{P}_{1}$.

The degree mixing was calculated at each plane $\left(\mathrm{P}_{n}\right)$ showing the result of each $n$th cycle. From the numerical solution of each solutes' concentration, the molar fraction of ligand $\left(x_{1}=\frac{c_{1}}{\sum_{i} c_{i}}, i=1, m\right.$, and $\left.\mathrm{Pt}\right)$ is used in order to quantify the

degree of mixing which directly denotes the progress of MOF nucleation and $\mathrm{Pt}$ reduction. The degree of mixing $(\varepsilon)$ is defined with its standard deviation of $x_{1}(\sigma)$, as below:

$$
\varepsilon=1-\frac{\sigma}{\sigma_{\max }}: \text { degree of mixing. }
$$

The maximum standard deviation $\left(\sigma_{\max }\right)$ was designated at the plane where the ligand or Pt precursor and metal meet first (i.e., $\sigma_{\max }=0.498$ for ligand-metal and 0.287 for ligand-Pt precursor at $\mathrm{P}_{1}$ ).

Fabrication of microfluidic blade. First, PI films of $125 \mu \mathrm{m}$ in thickness (Kapton HN film, Dupont, USA) were ablated using UV laser ( $355 \mathrm{~nm}$, ESI, USA) to form the desired micropatterns ( $300 \mu \mathrm{m}$ in width). In laser, ablated regions were completely eliminated from the PI films. Thereafter, FEP (fluoroethylene propylene) nanopowder dispersed in an aqueous solution was spin-coated (2000 rpm, $50 \mathrm{~s}$ ) onto each of the PI films. Then the FEP-coated PI films were vertically stacked using a metal holder with aligners. Finally, the aligned PI films were mechanically pressed at $350{ }^{\circ} \mathrm{C}$ under a pressure of $10 \mathrm{kPa}$ for $3 \mathrm{~h}$.

Synthesis of $\mathbf{C u}_{\mathbf{3}}(\mathbf{H H T P})_{2}$ film using MiCS process. A solution of Copper(II) acetate monohydrate $(0.12 \mathrm{M}$ in DMSO, $200 \mu \mathrm{L} / \mathrm{min})$ and a solution of HHTP $(0.1$ $\mathrm{M}$ in DMSO, $200 \mu \mathrm{L} / \mathrm{min}$ ) were introduced into two inlets of the microfluidic blade continuously using syringe pumps (Harvard Apparatus PHD 4400) at a rate of 400 $\mu \mathrm{L} / \mathrm{min}$. The microfluidic channels were shaped as a three-dimensional serpentine structure with a total of 15 cycles of mixing to induce effective mixing. The reacted solution is discharged through the 64-outlets of the microfluidic blade onto the heated substrate $\left(150^{\circ} \mathrm{C}\right)$. The gap (i.e., the distance between microfluidic blade and substrate) and angle were set at $100 \mu \mathrm{m}$ and $30^{\circ}$, respectively. The generated $\mathrm{Cu}_{3}(\mathrm{HHTP})_{2}$ thin-film was washed with DMSO and ethanol, respectively.

Synthesis of Pt@Cu $\mathrm{Cu}_{3}(\mathrm{HHTP})_{2}$ film using MiCS process. A solution of copper(II) acetate monohydrate $(0.12 \mathrm{M}$ in DMSO, $200 \mu \mathrm{L} / \mathrm{min})$ and a mixed solution of 
HHTP with $\mathrm{NaBH}_{4}$ (HHTP: $0.1 \mathrm{M}$ in DMSO, $\mathrm{NaBH}_{4}: 1 \mathrm{mg} \mathrm{mL}^{-1}, 200 \mu \mathrm{L} / \mathrm{min}$ ) were introduced to two inlets of the microfluidic blade using syringe pumps. $\mathrm{Pt}$ solution $\left(1 \mathrm{mg} \mathrm{mL}^{-1}, 50,100,150\right.$, and $\left.200 \mu \mathrm{L} / \mathrm{min}\right)$ was inserted at the 9 th cycle of mixing. The resulting solution is discharged continuously between the microfluidic blade and the heated substrate $\left(150^{\circ} \mathrm{C}\right)$. The gap (i.e., the distance between the microfluidic blade and substrate) and angle were set at $100 \mu \mathrm{m}$ and $30^{\circ}$, respectively. The generated Pt@ $\mathrm{Cu}_{3}(\mathrm{HHTP})_{2}$ thin-film was washed with DMSO and ethanol, respectively.

Material characterization. Cryo Field Emission TEM (Glacios, Thermo Fisher) at $200 \mathrm{kV}$, field-emission TEM (Tecnai G2 S-Twin, FEI) at $300 \mathrm{kV}$, and spherical aberration-corrected TEM (JEMARM200F, JEOL) at $200 \mathrm{kV}$ were conducted to investigate the microstructure of the samples. The MOF thin films were imaged using a field-emission scanning-electron microscope (FE-SEM, Hitachi S-4800). Xray diffraction (XRD) patterns were measured by D/Max-2500 (RIGAKU) diffractometer. Film thickness and topology were measured with tapping mode AFM (AFM WORKSHOP, PS-2010). The nitrogen adsorption-desorption isotherms were obtained using a BELSORP-max at $77 \mathrm{~K}$. Prior to the adsorption measurements, all samples were collected by a thick layer of MOF films $(\sim 20 \mathrm{mg})$ and were evacuated $\left(P<10^{-5} \mathrm{mbar}\right)$ at $393 \mathrm{~K}$ for $5 \mathrm{~h}$. To obtain $20 \mathrm{mg}$ of the samples using our method, we fabricated 15-20 thick films (shearing size: about $45 \times 60 \mathrm{~mm}$ ). The specific surface area was obtained by the Brunauer-Emmett-Teller (BET) method.

Sensing measurements. $\mathrm{Cu}_{3}(\mathrm{HHTP})_{2}$ and $\mathrm{Pt} @ \mathrm{Cu}_{3}(\mathrm{HHTP})_{2}$ thin films were directly formed on alumina $\left(\mathrm{Al}_{2} \mathrm{O}_{3}\right)$ substrate $(2.5 \mathrm{~mm}$ (width) $\times 2.5 \mathrm{~mm}$ (length) $\times$ $0.2 \mathrm{~mm}$ (thickness)). Bulk $\mathrm{Cu}_{3}(\mathrm{HHTP})_{2}$-based sensors were prepared by dropcoating suspension of the $\mathrm{Cu}_{3}(\mathrm{HHTP})_{2}$ powder $(5 \mathrm{mg}$ in $300 \mu \mathrm{L}$ of ethanol) onto the alumina substrate. To trace the resistance of the sensors, Au electrodes with a gap of $70 \mu \mathrm{m}$ were deposited on top of the films. $\mathrm{NO}_{2}$ sensing tests were carried out at room temperature in air. Before the sensing tests, the sensors were stabilized by fresh air for $4 \mathrm{~h}$. Thereafter, the sensors were exposed to $\mathrm{NO}_{2}$ for $20 \mathrm{~min}$, with the $\mathrm{NO}_{2}$ concentrations controlled in the range of 0.1-3 ppm using a mass flow controller. Resistance of the sensors was monitored using an acquisition system (34972, Agilent) in real time. The response was defined as a ratio of the resistance change $(\Delta R)$ to the resistance in the air $\left(R_{0}\right)$. Response time $\left(t_{90}\right)$ was the set as the time taken to reach $90 \%$ of the maximum resistance change $\left(0.9 \Delta R_{\max }\right)$.

\section{Data availability}

The authors declare that the data supporting the findings of this study are available within the article and its Supplementary Information files. Extra data are available from the corresponding author upon reasonable request.

Received: 21 December 2020; Accepted: 24 June 2021; Published online: 13 July 2021

\section{References}

1. Xie, L. S., Skorupskii, G. \& Dincă, M. Electrically conductive metal-organic frameworks. Chem. Rev. 120, 8536-8580 (2020).

2. Sun, L., Campbell, M. G. \& Dinca, M. Electrically conductive porous metalorganic frameworks. Angew. Chem. Int. Ed. 55, 3566-3579 (2016).

3. Hmadeh, M. et al. New porous crystals of extended metal-catecholates. Chem. Mater. 24, 3511-3513 (2012).

4. Huang, X. et al. A two-dimensional pi-d conjugated coordination polymer with extremely high electrical conductivity and ambipolar transport behaviour. Nat. Commun. 6, 7408 (2015)

5. Ko, M., Mendecki, L. \& Mirica, K. A. Conductive two-dimensional metalorganic frameworks as multifunctional materials. Chem. Commun. 54, 7873-7891 (2018).

6. Wu, G., Huang, J., Zang, Y., He, J. \& Xu, G. Porous field-effect transistors based on a semiconductive metal-organic framework. J. Am. Chem. Soc. 139, 1360-1363 (2017).

7. Sheberla, D. et al. Conductive MOF electrodes for stable supercapacitors with high areal capacitance. Nat. Mater. 16, 220-224 (2017).

8. Li, W.-H. et al. Conductive metal-organic framework nanowire array electrodes for high-performance solid-state supercapacitors. Adv. Funct. Mater. 27, 1702067 (2017).

9. Feng, D. et al. Robust and conductive two-dimensional metal-organic frameworks with exceptionally high volumetric and areal capacitance. Nat. Energy 3, 30-36 (2018).

10. Nam, K. W. et al. Conductive 2D metal-organic framework for highperformance cathodes in aqueous rechargeable zinc batteries. Nat. Commun. 10, 4948 (2019).
11. Koo, W.-T., Jang, J.-S. \& Kim, I.-D. Metal-organic frameworks for chemiresistive sensors. Chem 5, 1938-1963 (2019).

12. Campbell, M. G., Liu, S. F., Swager, T. M. \& Dincă, M. Chemiresistive sensor arrays from conductive 2D metal-organic frameworks. J. Am. Chem. Soc. 137, 13780-13783 (2015)

13. Smith, M. K. \& Mirica, K. A. Self-organized frameworks on textiles (SOFT): conductive fabrics for simultaneous sensing, capture, and filtration of gases. J. Am. Chem. Soc. 139, 16759-16767 (2017).

14. Yao, M. S. et al. Layer-by-layer assembled conductive metal-organic framework nanofilms for room-temperature chemiresistive sensing. Angew. Chem. Int. Ed. 56, 16510-16514 (2017).

15. Koo, W.-T., Kim, S.-J., Jang, J.-S., Kim, D.-H. \& Kim, I.-D. Catalytic metal nanoparticles embedded in conductive metal-organic frameworks for chemiresistors: highly active and conductive porous materials. Adv. Sci. 6 , 1900250 (2019).

16. Choi, K. M., Na, K., Somorjai, G. A. \& Yaghi, O. M. Chemical environment control and enhanced catalytic performance of platinum nanoparticles embedded in nanocrystalline metal-organic frameworks. J. Am. Chem. Soc. 137, 7810-7816 (2015).

17. Wei, S. et al. Direct observation of noble metal nanoparticles transforming to thermally stable single atoms. Nat. Nanotechnol. 13, 856-861 (2018).

18. Li, G., Zhao, S., Zhang, Y. \& Tang, Z. Metal-organic frameworks encapsulating active nanoparticles as emerging composites for catalysis: Recent progress and perspectives. Adv. Mater. 30, 1800702 (2018).

19. Aulakh, D. et al. Direct imaging of isolated single-molecule magnets in metalorganic frameworks. J. Am. Chem. Soc. 141, 2997-3005 (2019).

20. Cai, D. et al. A highly conductive MOF of graphene analogue $\mathrm{Ni}_{3} \mathrm{HITP}_{2}$ as a sulfur host for high-performance lithium-sulfur batteries. Small 15, 1902605 (2019).

21. Hoppe, B. et al. Graphene-like metal-organic frameworks: morphology control, optimization of thin film electrical conductivity and fast sensing applications. CrystEngComm 20, 6458-6471 (2018).

22. Song, X. et al. 2D semiconducting metal-organic framework thin films for organic spin valves. Angew. Chem. Int. Ed. 59, 1118-1123 (2020).

23. Rubio-Gimenez, V. et al. Bottom-up fabrication of semiconductive metalorganic framework ultrathin films. Adv. Mater. 30, 1704291 (2018).

24. Yuan, K. et al. Construction of large-area ultrathin conductive metal-organic framework films through vapor-induced conversion. Small 15, 1804845 (2019).

25. Meilikhov, M. et al. Metals@MOFs-loading MOFs with metal nanoparticles for hybrid functions. Eur. J. Inorg. Chem. 24, 3701-3714 (2010).

26. Yu, J. et al. Nanoparticle/MOF composites: preparations and applications. Mater. Horiz. 4, 557-569 (2017).

27. Campbell, M. G., Sheberla, D., Liu, S. F., Swager, T. M. \& Dinca, M. $\mathrm{Cu}_{3}$ (hexaiminotriphenylene) ${ }_{2}$ : an electrically conductive $2 \mathrm{D}$ metal-organic framework for chemiresistive sensing. Angew. Chem. Int. Ed. 54, 4349-4352 (2015).

28. Lee, J.-C., Kim, J.-O., Lee, H.-J., Shin, B. \& Park, S. Meniscus-guided control of supersaturation for the crystallization of high quality metal organic framework thin films. Chem. Mater. 31, 7377-7385 (2019).

29. Giri, G. et al. Tuning charge transport in solution-sheared organic semiconductors using lattice strain. Nature 480, 504-508 (2011).

30. Kim, J.-O. et al. Inorganic polymer micropillar-based solution shearing of large-area organic semiconductor thin films with pillar-size-dependent crystal size. Adv. Mater. 30, 1800647 (2018).

31. Echaide-Górriz, C., Clément, C., Cacho-Bailo, F., Téllez, C. \& Coronas, J. New strategies based on microfluidics for the synthesis of metal-organic frameworks and their membranes. J. Mater. Chem. A 6, 5485-5506 (2018).

32. Faustini, M. et al. Microfluidic approach toward continuous and ultrafast synthesis of metal-organic framework crystals and hetero structures in confined microdroplets. J. Am. Chem. Soc. 135, 14619-14626 (2013).

33. Wang, Y. et al. Bottom-up fabrication of ultrathin $2 \mathrm{D} \mathrm{Zr}$ metal-organic framework nanosheets through a facile continuous microdroplet flow reaction. Chem. Mater. 30, 3048-3059 (2018).

34. Kim, H. et al. Submillisecond organic synthesis: Outpacing Fries rearrangement through microfluidic rapid mixing. Science 352, 691-694 (2016).

35. Gu, X., Shaw, L., Gu, K., Toney, M. F. \& Bao, Z. The meniscus-guided deposition of semiconducting polymers. Nat. Commun. 9, 534 (2018).

36. Hao, J. et al. Synthesis of high-performance polycrystalline metal-organic framework membranes at room temperature in a few minutes. J. Mater. Chem. A 8, 7633-7640 (2020).

37. Suryawanshi, P. L., Gumfekar, S. P., Kumar, P. R., Kale, B. B. \& Sonawane, S. H. Synthesis of ultra-small platinum nanoparticles in a continuous flow microreactor. Colloid Interface Sci. Commun. 13, 6-9 (2016).

38. Le Berre, M., Chen, Y. \& Baigl, D. From convective assembly to LandauLevich deposition of multilayered phospholipid films of controlled thickness. Langmuir 25, 2554-2557 (2009). 
39. Potyrailo, R. A. Multivariable sensors for ubiquitous monitoring of gases in the era of internet of things and industrial internet. Chem. Rev. 116, 11877-11923 (2016).

40. Kim, S.-J., Choi, S.-J., Jang, J.-S., Cho, H.-J. \& Kim, I.-D. Innovative nanosensor for disease diagnosis. Acc. Chem. Res. 50, 1587-1596 (2017).

41. Loutfi, A., Coradeschi, S., Mani, G. K., Shankar, P. \& Rayappan, J. B. B. Electronic noses for food quality: a review. J. Food Eng. 144, 103-111 (2015).

42. Rubio-Giménez, V. et al. Origin of the chemiresistive response of ultrathin films of conductive metal-organic frameworks. Angew. Chem. Int. Ed. 130, 15306-15310 (2018).

43. Choi, S.-J. et al. Metal-organic framework-templated $\mathrm{PdO}-\mathrm{Co}_{3} \mathrm{O}_{4}$ nanocubes functionalized by SWCNTs: improved $\mathrm{NO}_{2}$ reaction kinetics on flexible heating film. ACS Appl. Mater. Interfaces 9, 40593-40603 (2017).

44. Rui, K. et al. Dual-function metal-oganic framework-based wearable fibers for gas probing and energy storage. ACS Appl. Mater. Interfaces 10, 2837-2842 (2018).

45. Chen, Z. et al. Mimicking a dog's nose: scrolling graphene nanosheets. ACS Nano 12, 2521-2530 (2018).

46. Yuan, W., Liu, A., Huang, L., Li, C. \& Shi, G. High-performance $\mathrm{NO}_{2}$ sensors based on chemically modified graphene. Adv. Mater. 25, 766-771 (2013).

47. Yang, W. et al. Additive-free synthesis of $\operatorname{In}_{2} \mathrm{O}_{3}$ cubes embedded into graphene sheets and their enhanced $\mathrm{NO}_{2}$ sensing performance at room temperature. ACS Appl. Mater. Interfaces 6, 21093-21100 (2014).

48. Cho, B. et al. Charge-transfer-based gas sensing using atomic-layer $\mathrm{MoS}_{2}$. Sci. Rep. 5, 8052 (2015).

49. Ko, K. Y. et al. Improvement of gas-sensing performance of large-area tungsten disulfide nanosheets by surface functionalization. ACS Nano 10, 9287-9296 (2016).

50. Xu, T. et al. The ultra-high $\mathrm{NO}_{2}$ response of ultra-thin $\mathrm{WS}_{2}$ nanosheets synthesized by hydrothermal and calcination processes. Sens. Actuators B 259, 789-796 (2018).

51. Jaiswal, J., Sanger, A., Tiwari, P. \& Chandra, R. $\mathrm{MoS}_{2}$ hybrid heterostructure thin film decorated with CdTe quantum dots for room temperature $\mathrm{NO}_{2}$ gas sensor. Sens. Actuators B: Chem. 305, 127437 (2020).

52. Zhou, Y., Liu, G., Zhu, X. \& Guo, Y. Ultrasensitive $\mathrm{NO}_{2}$ gas sensing based on $\mathrm{rGO} / \mathrm{MoS}_{2}$ nanocomposite film at low temperature. Sens. Actuators B 251, 280-290 (2017).

\section{Acknowledgements}

This research has been supported by the POSCO Science Fellowship of POSCO TJ Park Foundation; the Brain Korea21FOUR(BK21FOUR)(4199990514310); the National Research Foundation of Korea (NRF) grant funded by the Korea government (MSIT) (2019R1A5A8080326); the Technology Innovation Program (no. 20012463) funded by the Ministry of Trade, Industry \& Energy (MOTIE) of the Republic of Korea; a grant from the Korea Institute of Energy Technology Evaluation and Planning (KETEP) and the Ministry of Trade Industry \& Energy (MOTIE) of the Republic of Korea (no. 20183010014470); Nano Convergence Foundation funded by the Ministry of Science and ICT (MSIT, Korea) and the Ministry of Trade, Industry, and Energy (MOTIE,
Korea) (project no.: 170720000230); and National Research Foundation (NRF) of the Korean Government (MSIT) (2020R1A2C301312711).

\section{Author contributions}

J.-O.K. and W.-T.K. conceived the concept and designed experiments. J.-O.K. fabricated the polyimide microfluidic device, MOF thin films, and conducted structural characterization. W.-T.K. conducted sensing tests. H.K. and S.C. conducted CFD simulation and analysis with J.-O.K. C.P. assisted sensing measurements. T.L., C.A.H., and D.S.K. assisted MOF film fabrications. All authors contributed to the writing of the manuscript. All authors discussed the results and the manuscript. I.D.K. and S.P. were responsible for managing all aspects of this project.

\section{Competing interests}

The authors declare no competing interests.

\section{Additional information}

Supplementary information The online version contains supplementary material available at https://doi.org/10.1038/s41467-021-24571-1.

Correspondence and requests for materials should be addressed to I.-D.K. or S.P.

Peer review information Nature Communications thanks Roland Fischer, Stefan Wuttke and the other, anonymous, reviewer(s) for their contribution to the peer review of this work. Peer reviewer reports are available.

Reprints and permission information is available at http://www.nature.com/reprints

Publisher's note Springer Nature remains neutral with regard to jurisdictional claims in published maps and institutional affiliations.

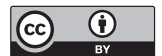

Open Access This article is licensed under a Creative Commons Attribution 4.0 International License, which permits use, sharing, adaptation, distribution and reproduction in any medium or format, as long as you give appropriate credit to the original author(s) and the source, provide a link to the Creative Commons license, and indicate if changes were made. The images or other third party material in this article are included in the article's Creative Commons license, unless indicated otherwise in a credit line to the material. If material is not included in the article's Creative Commons license and your intended use is not permitted by statutory regulation or exceeds the permitted use, you will need to obtain permission directly from the copyright holder. To view a copy of this license, visit http://creativecommons.org/ licenses/by/4.0/.

(C) The Author(s) 2021 